\title{
Was There a Negative Vacuum Energy in Your Past?
}

\author{
George Chapline1, James Barbieri² \\ ${ }^{1}$ Lawrence Livermore National Laboratory, Livermore, CA, USA \\ ${ }^{2}$ Retired, Naval Air Warfare Center, China Lake, CA, USA \\ Email: chapline1@llnl.gov, barbierijf@hughes.net
}

How to cite this paper: Chapline, G. and Barbieri, J. (2019) Was There a Negative Vacuum Energy in Your Past? Journal of Modern Physics, 10, 1166-1176.

https://doi.org/10.4236/jmp.2019.1010077

Received: June 24, 2019

Accepted: August 27, 2019

Published: August 30, 2019

Copyright $\odot 2019$ by author(s) and Scientific Research Publishing Inc. This work is licensed under the Creative Commons Attribution International License (CC BY 4.0).

http://creativecommons.org/licenses/by/4.0/

\begin{abstract}
We introduce a novel model for the origin of the observable universe in which a flat universe with a positive vacuum energy is proceeded by a flat universe with a negative vacuum energy. A negative vacuum energy is consistent with a supersymmetric ground state similar to that predicted by superstring theories. A positive vacuum energy could emerge as a result of the gravitational collapse of the negative vacuum energy universe when the matter temperature reaches a characteristic value where supersymmetry is strongly broken. In principle this allows one to derive all the features of our expanding universe from a single parameter: the magnitude of the pre-big bang negative vacuum energy density. In this paper, a simple model for the big bang is introduced which allows us to use the present day entropy density, and temperature fluctuations of the $\mathrm{CMB}$, together with the present day density of dark matter, to predict the magnitude of the negative vacuum energy. This model for the big bang also makes a dramatic prediction: dark matter consists of compact objects with masses on the order of $10^{4}$ solar masses. Remarkably this is consistent with numerical simulations for how the primordial fluctuations in the density of dark matter give rise to the observed inhomogeneous distribution of matter in our universe. Our model for the big bang also allows for the production of some compact objects with masses greater than $10^{4}$ solar masses which are consistent with observations of massive compact objects at the center of the earliest galaxies.
\end{abstract}

\section{Keywords}

Gravitational Collapse, Cosmology, Dark Matter, Vacuum Energy, CMB

\section{Introduction}

One of the outstanding puzzles of modern theoretical physics is that classical 
general relativity offers no clue as the fate of matter undergoing gravitational collapse or the state of matter prior to the "big bang". These puzzles are all the more perplexing because in quantum mechanics it is not possible for matter to simply appear or disappear. Previously we have drawn attention [1] [2] to the fact that the quantum critical phase transition theory of event horizons [3] provides a plausible explanation for the fate of matter undergoing gravitational collapse; namely most of the mass-energy is converted into vacuum energy resulting in the formation of a "dark energy star" [4]. Dark energy stars are distinguished from black holes in that their interiors resemble deSitter or Godel "interior" solutions [5] rather than a black hole space-times predicted by classical general relativity. In this paper, we offer a possible resolution of the enigma of what preceded the big bang by noting that a flat Robertson-Walker universe with a negative cosmological constant will naturally evolve via the same kind of quantum dynamics that resolves the problem of gravitational collapse to an expanding inhomogeneous universe containing radiation and dark matter. It was suggested some time ago by deSitter, Eddington, and Lemaitre [6] that the observable universe may not have had a singular beginning, but, instead may have originated from a finite size seed. Lemaitre suggested that this finite seed was a macroscopic quantum state which he called the "primeval atom". Cosmological models incorporating this idea make use of Lemaitre's examples of Robertson-Walker space-times with positive cosmological constant [7] [8]. In the following we describe a model for the origin of the expanding universe, in which the initial state of the observable universe is not a single quantum object, but an infinite assembly of quantum objects. It has already been noted [9] that such a two-phase cosmology provides a simple explanation for many of the observed features of our universe, including the entropy and temperature fluctuations of the cosmic microwave background. In this paper we describe how a 2-phase model for the initial state of our expanding universe can arise from the gravitational collapse of a flat Robertson-Walker universe with a negative cosmological constant. We also indicate how the parameters of the standard cosmological model as well the present day large scale inhomogeneous structure of our universe might be derived from a single parameter: the magnitude of the initial negative vacuum energy.The classical gravitational dynamics of a flat universe with a negative cosmological constant necessarily involves collapse to a density singularity. The acceleration of of the cosmological scale factor $R(t)$ in a flat Robertson-Walker universe with a cosmological constant is

$$
\ddot{R}=-\frac{4 \pi G}{3 c^{2}}\left(\rho+3 p-2 \rho_{\Lambda}\right) R^{2}
$$

where $\rho$ is the matter density, $p$ is the matter pressure and $\rho_{\Lambda}$ is the vacuum energy density. When the vacuum energy density $\rho_{\Lambda}$ is negative and the matter is a relativistic gas of particles with an adiabatic index $4 / 3$, Equation (1) has a simple analytic solution [8]:

$$
R(\tau)=R_{m}\left[\frac{1-\cos 4 \alpha \tau}{2}\right]^{1 / 4}
$$


where $\tau$ is the usual Robertson-Walker universal time. The cosmological constant $\Lambda=-3 \alpha^{2}=8 \pi G \rho_{\Lambda} / c^{2}$. Regardless of it's maximum value the scale collapses to zero in a time $\tau_{c} \equiv \pi / 4 \alpha$. At the time $\tau=\pi / 4 \alpha$ when the scale factor is a maximum the total energy density $\rho+\rho_{\Lambda}=0$. As $\tau$ approaches $\pi / 2 \alpha$ the energy density which is dominated by the matter density $\rho$ approaches infinity. In Figure 1, we show the evolution of the scale factor for an initial scale factor $R_{m}=10 R_{g}$ where $R_{g}$ is the initial gravitational radius for the matter. We also show the light sphere radius $r_{c}$ for photons emitted at the initial time $\tau=\pi / 4 \alpha$. Equation (2). implies that a conformal radius

$$
r_{c} / R=\sqrt{2} R_{g} / R_{m} F\left(\cos ^{-1}\left(R / R_{m}\right) \mid \pi / 4\right)
$$

where $F$ is an incomplete elliptic integral of the first kind. As is evident from Figure 1, photons emitted from any point in the negative cosmological constant universe are trapped, and according to Penrose and Hawking would require collapse to a singularity. On the other hand, we will assume that in reality a negative cosmological constant does not collapse to a singularity due to quantum effects.

\section{Model for the Big Bang}

Our hypothesis is that the same type of conversion of matter mass-energy to vacuum energy [10] that we previously been proposed [1] [2] as the reason for the avoidance of a singular end point for the gravitational collapse of massive stellar cores will also lead to the avoidance of a mass density singularity in a flat

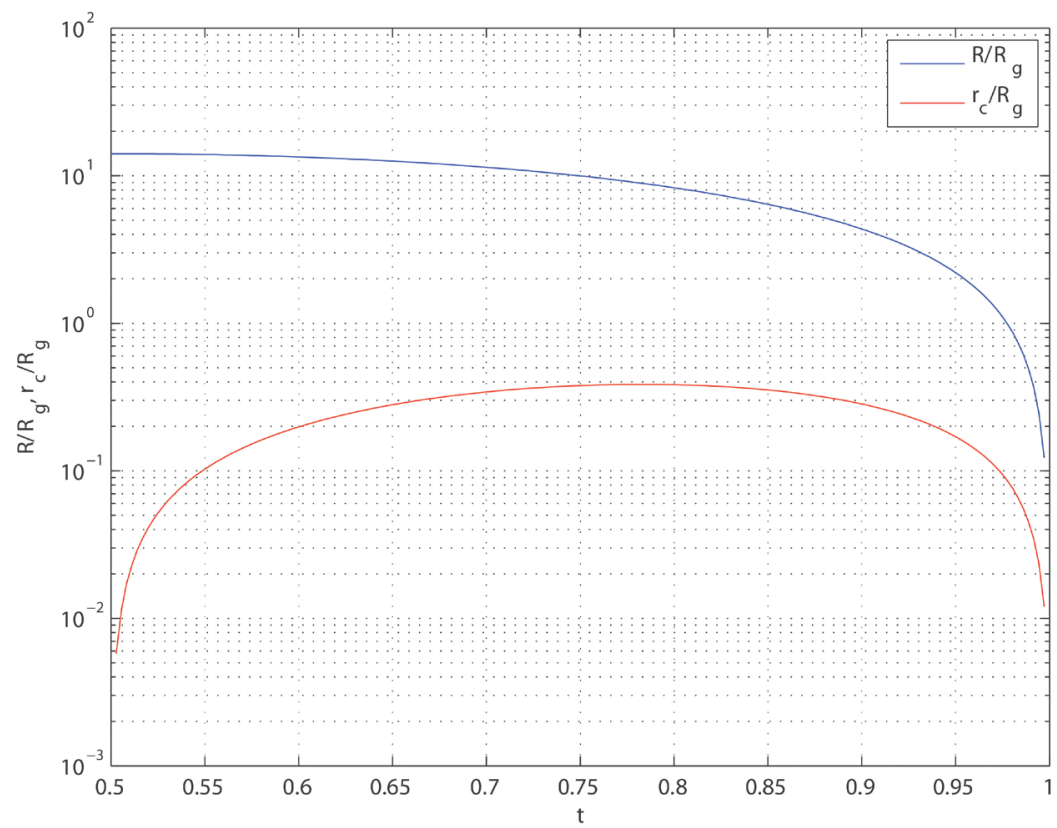

Figure 1. Time evolution of the scale factor in a radiation filled flat Robertson-Walker universe with a negative cosmological constant, together with the light sphere radius for photons emitted at the initial time $t=0.5$. Time is measure in units of $\pi / 2 \alpha$, while the radii are measured in units of the initial matter gravitational radius $C / \alpha$. 
negative cosmological constant universe. In particular, we will argue that as a result of the ubiquitous formation of trapped surfaces in a flat negative cosmological constant universe most of the matter mass-energy will be transformed into positive vacuum energy, resulting in an expanding universe which resembles our universe. As a simple model for the conversion of most of the mass-energy of radiation in our negative cosmological constant universe to vacuum energy we propose replacing the usual conservation law for a Lemaitre universe with a constant cosmological constant with the equations

$$
\begin{gathered}
\frac{\mathrm{d}}{\mathrm{d} t}\left(\rho R^{3}\right)+p \frac{\mathrm{d} R^{3}}{\mathrm{~d} t}=-\frac{\rho R^{3}}{\tau_{c}} \\
\frac{\mathrm{d} \rho_{\Lambda}}{\mathrm{d} t}=\frac{\rho}{\tau_{c}}
\end{gathered}
$$

Numerical solutions of Equations (1), (3) and (4) are shown in Figure 2 and Figure 3. It can be seen that the acceleration of the Robertson-Walker scale factor switches from being negative to positive, indicating evolution from a collapsing to an expanding universe. Our model for the big bang consists of Equations (1), (3) and (4) together with the stipulation that after a time $\tau_{c}=\pi / 2 \alpha$ the vacuum energy created when the deSitter horizon is small compared to the Hubble radius does not contribute to a cosmological constant, but instead is encapsulated into a form of dark matter.

Of course the ultimate fate of matter undergoing gravitational collapse has been a long standing enigma. Following the seminal paper of Oppenheimer and Snyder, it had come to be widely accepted that the gravitational collapse of a sufficiently large mass would inevitably lead to the formation of an event horizon and a density singularity [11]. Moreover, it has generally been believed

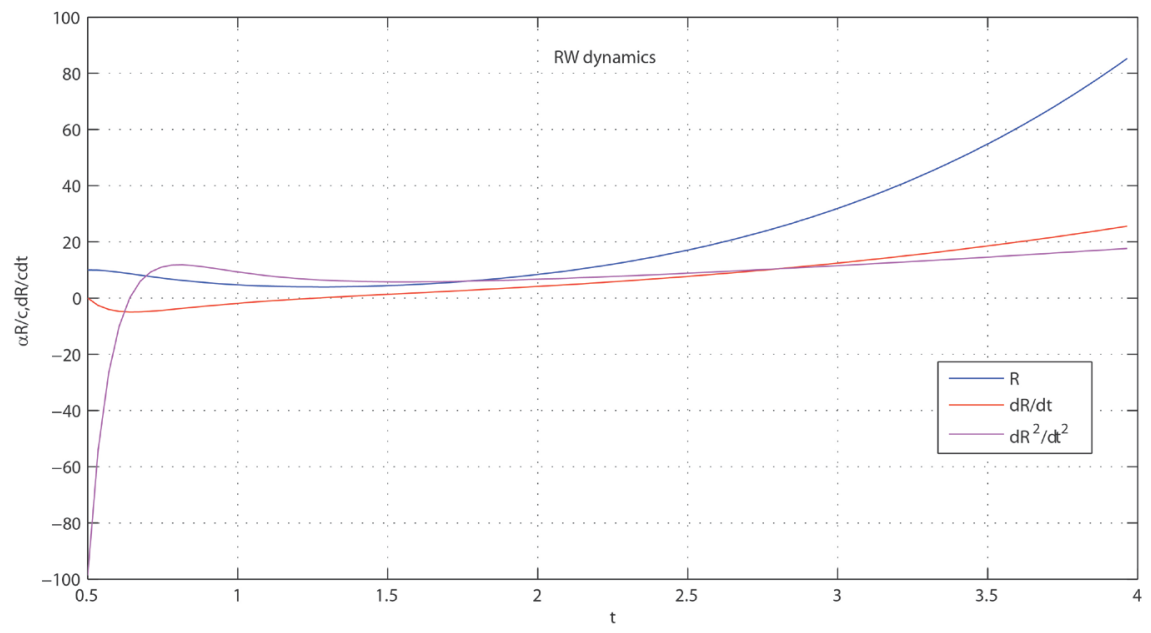

Figure 2. Evolution of a flat Robertson-Walker universe, initially with a negative cosmological constant and filled with radiation, but allowing for the radiation and vacuuum energy density to change according to Equations (3) and (4). Time is measured in units of $\pi / 2 \alpha$ while the radii are measured in units of the initial matter gravitational radius $R_{g}=C / \alpha$. 


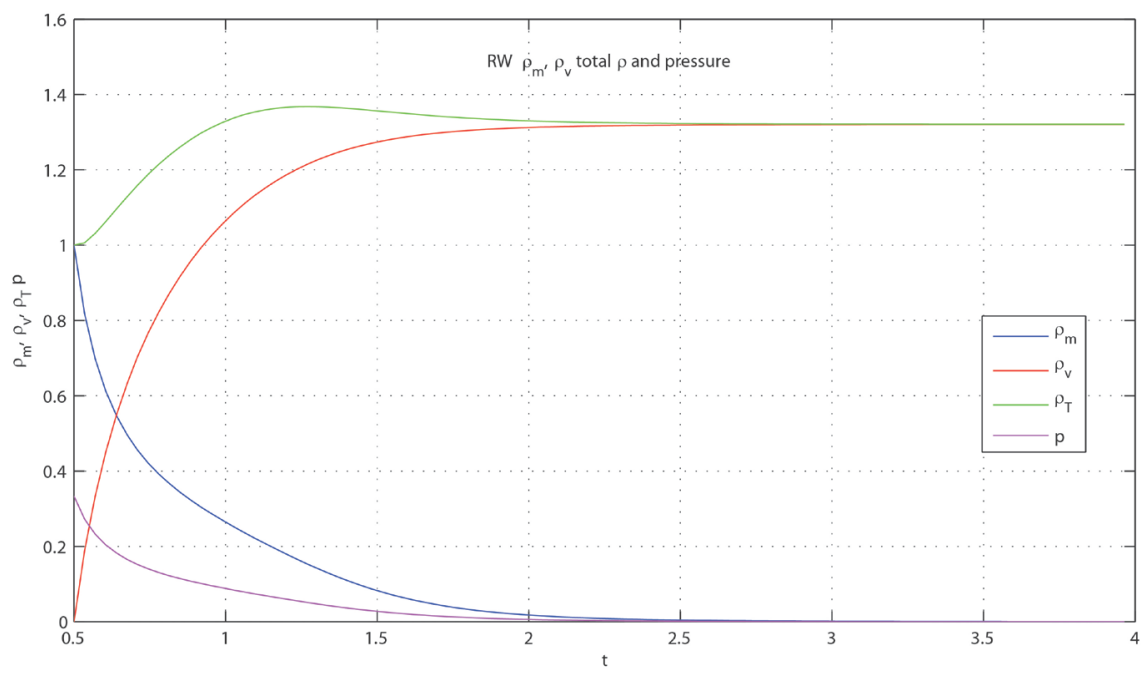

Figure 3. The matter, vacuum and total energy densities resulting from the collapse of a flat negative vacuum energy universe.

that these predictions will turn out to be correct even when quantum effects are taken into account, since the formation of an event horizon can take place in a region of space-time where the curvature is very small. On the other hand, there are several long standing puzzles connected with general relativistic picture of gravitational collapse. The most famous of these puzzles concerns the fact that in quantum mechanics information can never disappear. The most likely resolution of this paradox is that quantum effects profoundly affect the classical picture of matter in-falling smoothly through an event horizon. In particular, there are plausible arguments [3] [12] [13] that in a quantum theory of gravity the space-time inside an event horizon always resembles deSitter's "interior" solution of the Einstein equations [5].

\section{Estimate of the Mass Range for Dark Matter MACHOs}

A central central element for our argument that a negative cosmological constant evolves into an expanding universe that resembles our own is that, due to the well known instability of infinite deSitter space at the deSitter horizon [3], patches of space-time resembling deSitter interior solutions will appear throughout the collapsing universe. These "dark energy stars" are gravitationally stable, and will have a mass

$$
M^{*}=0.3\left[(\mathrm{GeV})^{4} / \rho^{*}\right]^{1 / 2} M_{\odot}
$$

where $\rho^{*}$ is the positive vacuum energy created at the collapse time $\tau_{c}=\pi / 2 \alpha$ by the conversion of radiation energy in the collapsing negative cosmological universe into vacuum energy and $M_{\odot}$ is the mass of the sun. This mass is just the mass inside the deSitter horizon at the time $\tau_{c}$. The two-phase picture for cosmology [14] where space-time is a mixture of ordinary vacuum and dark energy stars, emerges from our model in somewhat the same way that supersaturated steam consists of a mixture of water vapor and water droplets. It 
is of course rather natural to imagine that in such a picture the initial energy densities of the dark matter and the cosmological vacuum may be comparable. The initial masses of the dark energy stars will be given by Equation (6) but because the spatial density of these dark energy stars will be very large, collisions and fluctuations in the spatial density of the primordial dark energy stars created at $\tau_{c}=\pi / 4 \alpha$ will cause them coalesce, (the details are discussed in Ref. [9]) leading to the formation of more massive compact objects. The reversal of the scale factor acceleration from negative to positive will result in a universe consisting of dark energy stars and radiation expanding in a Freidmann-like fashion. The maximum mass of these compact objects will be dictated by the time it takes for their spatial density to become too low for them to continue to coalesce (the details are discussed in Ref. [9]). We are immediately faced with the puzzle though that the expansion of a cloud of dark energy stars with an initial mass-energy density of $\rho^{*}$ would lead to a present day density of matter that is many orders of magnitude greater than the observed matter density.

A possible resolution of this puzzle [9] is that when is that when the dark energy stars coalesce the surface area of the larger dark energy stars will be maximized in much the same a way that the total that the total surface area increases when two black holes coalesce. Because of this black hole-like behavior a large fraction of the mass-energy of dark energy stars is converted into thermal energy when they coalesce. Our model for the big bang is based on the assumption that this thermal radiation is released as freely as the streaming radiation when the photon frequency falls below a critical frequency $v_{c}$ where the radiation and the dark energy stars decouple. The value of this critical frequency was estimated in Ref.s [1] [2] [3]. If we assume that the gauge field coupling strength at the GUT scale $g^{2}=0.1$, this estimate for cutoff for strong interactions between dark energy stars and photons is $h v_{c}=1 \mathrm{GeV}\left(M_{\odot} / M\right)^{1 / 2}$ where $M$ is the mass of the dark energy star.

In our model for the big bang transition between the very high temperature regime where there is strong coupling between the dark matter and radiation and the lower temperature regime where the dark matter and radiation are decoupled is assumed to be abrupt in the sense that for red shifts greater than certain red shift $1+z_{r}$, the radiation energy is stored as the mass-energy of dark energy stars with masses $M \gg M^{*}$, while for $1+z<1+z_{r}$ we will assume that all the mass-energy of the primordial dark energy stars will have been converted into radiation and remanent dark energy stars with average mass $M_{D M}$. Taking into account the black hole-like relation between mass and surface area of a dark energy star the cosmological energy density of the dark matter as a function of red shift following the decoupling with radiation for $1+z<1+z_{r}$ will be

$$
\rho_{D M}=\rho_{*}\left(\frac{M^{*}}{M_{D M}}\right)^{2}\left(\frac{1+z}{1+z_{*}}\right)^{3}
$$

where $M_{D M}$ is the average mass of the dark energy star and $1+z_{*}$ is the red shift for the break-up of the initial positive vacuum energy state resulting from the collapse of the negative vacuum energy state, corresponding to the origin of 
the observable universe. As an estimate for the red shift separating these two regimes we will use the value

$$
1+z_{r}=\frac{0.37 h v_{c}}{k_{B} T_{C M B}}
$$

where the factor 0.37 accounts for the difference between the temperature and the mean photon energy and $T_{C M B}=2.73 \mathrm{~K}$ is the present day temperature of the CMB. The radiation energy density for $1+z<1+z_{r}$ will be given by

$$
\rho_{r a d}=\rho_{*}\left(\frac{1+z_{*}}{1+z_{r}}\right)\left(\frac{1+z}{1+z_{*}}\right)^{4}
$$

The radiation energy density is related to the radiation temperature $T$ by the usual formula

$$
\rho_{\text {rad }}=N(T) \frac{\pi^{2}}{30} \frac{\left(k_{B} T\right)^{4}}{(\hbar c)^{3}}
$$

where $N(T)$ is the effective number of elementary particle species contributing to the radiation energy density at red shift $1+z$. Strictly speaking we should have taken into account $N(T)$ in our estimate, Equation (5), for the red shift marking the appearance of the $\mathrm{CMB}$, but we have neglected this correction since it only depends on $N^{1 / 4}$.

Combining Equations (6)-(8) with the ratio of the present day mass-energy densities of dark matter $\left(\mathrm{keV} / \mathrm{cm}^{3}\right)$ and the $\mathrm{CMB}\left(0.26 \mathrm{eV} / \mathrm{cm}^{3}\right)$ leads to the following relation between $M_{D M}$ and $M^{*}$ :

$$
\left(\frac{M_{D M}}{M_{\odot}}\right)^{5 / 4}=2 \times 10^{4}\left(\frac{M^{*}}{M_{\odot}}\right)
$$

Since in our model $M^{*}$ is unconstrained, Equation (10) formally allows the transition from a dark energy star dominated universe to a radiation dominated universe to take place for any for $M_{D M}$. However this transition cannot occur so late that it interferes with the requirement that the cosmological production of of helium and other light elements hould be approximately the same as in the standard cosmological model. This limits the value of the transition red shift $1+z_{r}$ to be $>10^{10}$ and $M_{D M}<2 \times 10^{4} M_{\odot}$. One may also invoke the limits on the present day abundance of $\mathrm{MACHO}$ objects set by gravitational micro-lensing [15] to say that $M_{D M}$ should be $>10 M_{\odot}$. In the following we will adopt as our apriori range for the average primordial compact object mass

$2 \times 10^{4} M_{\odot}>M_{D M}>10 M_{\odot}$. For these nominal values of the dark matter masses the $\mathrm{CMB}$ originates at a red shift in the range $5 \times 10^{11}>1+z_{r}>10^{10}$. The radiation temperature at redshift $z_{r}$ would lie in the range $120 \mathrm{MeV}>T\left(z_{r}\right)>2.6 \mathrm{MeV}$ which for the most part is above the temperature where the cosmological production of the light elements takes place

Equation (10) implies that our assumed range of dark matter masses the range of the initial primordial dark energy stars lie in the range $12 M_{\odot}>M^{*}>9 \times 10^{-4} M_{\odot}$. The initial positive vacuum energy density $\rho^{*}$ is 
related to $M^{*}$ by $\rho^{*}=0.1(\mathrm{GeV})^{4}\left(M_{\odot} / M^{*}\right)^{2}$, which just expresses the fact that for a dark energy star $M$ is the mass of the vacuum energy inside the deSitter horizon. The limits on $M^{*}$ derived from Equation (10) translates to $10^{5}(\mathrm{GeV})^{4}>\rho^{*}>7 \times 10^{-4}(\mathrm{GeV})^{4}$. Given the present day cosmological density of matter $\left(2 \times 10^{-30} \mathrm{gm} / \mathrm{cc}\right)$ the redshift where the dark energy stars were initially formed can be found from Equation (6) and lie in the range

$5 \times 10^{11}>1+z_{*}>210^{10}$ for our nominal range for $M_{D M}$. By construction the range for $M^{*}$ and $\rho^{*}$ just quoted are consistent with the present day density of dark matter. Our predicted present day mass spectrum extends to "intermediate masses" >1000M $\odot$ [16].

Equation (8) also yields a present day radiation temperature that is very close to the observed CMB temperature for all values of $M_{D M}$ in our nominal range. Another very encouraging prediction of our model follows from the fact that the metric fluctuations created by the quantum instability of the positive vacuum energy state created by the big bang at the deSitter horizons have the HarrisonZeldovich-Peebles form [17] [18] [19]:

$$
\frac{\delta \rho}{\rho} \approx \epsilon_{0}\left(R_{0} k\right)^{2}
$$

where $R_{0}=2 G M^{*} / c^{2}$ is the gravitational radius corresponding to the initial positive vacuum energy, $\epsilon_{0} \sim 1$ is the metric fluctuation created on the scale $R_{0}$ by the formation of the objects with mass $M^{*}$ and $\delta \rho / \rho$ is the fractional density fluctuation for scales $k^{1} \gg R_{0}$. Because the speed of sound in an expanding universe of dark energy stars is very low, the density fluctuations will rapidly grow until the radiation is locked up the as the energy excited dark energy star becomes freely streaming. According to the Lifschitz formula [20] for the growth of the density fluctuations during a matter dominated period by the time the red shift reaches $1+z_{r}$ the fluctuations in the density of primordial dark energy stars with mass $M^{*}$ will have grown by a factor $\left(1+z_{*} / 1+z_{r}\right)$ independent of length scale. Taking this into account and averaging the density fluctuations as predicted by Equation (11) over all volumes that could have been have collapses by the time that the expanding universe had reached the beginning of the radiation dominated era at red shift $1+z_{r}$, we obtain (see Ref. [9] for details) as an estimate of the renormalized value of $\varepsilon_{0}$ at red shift $1+z_{r}$ :

$$
\epsilon_{r} \approx 3 \epsilon_{0}\left[\frac{1+z_{r}}{1+z_{*}}\right]^{2}
$$

For our assumed range of dark matter average masses our model for the big bang predicts

$$
1.2 \times 10^{-5}>\left(\frac{1+z_{r}}{1+z_{*}}\right)^{2}>10^{-6}
$$

Considering the simplicity of our model these values are in remarkably good agreement with the observed value, $\delta T / T \sim 10^{-5}$, for the mean temperature 
fluctuation of the $\mathrm{CMB}$, which corresponds to $\epsilon_{0} \sim 3 \times 10^{-5}$. Taken literally Equation (12) suggests that the average dark matter compact object has a mass close to $10^{4} M_{\odot}$.

It is of course a dramatic prediction of our model that dark matter consists of compact objects with masses on the order of $10^{4} M_{\odot}$. Actually it is an old idea that dark matter consist of primordial black holes (PBHs) [21], although at the time this was proposed there was no preference for the typical masses of PBHs. Recently the idea that dark matter consists of compact objects has received renewed interest as a result of the failure to identify any stable elementary particles that might serve as a candidate for dark matter [22] [23] [24] [25]. Finally, it is certainly news worthy that our hypothesis that the initial vacuum energy was negative is consistent with superstring models for a supersymmetric ground state [16] [26].

\section{Conclusion}

Evidently, all of the features of the CMB as well as many features of dark matter follow from our hypothesis that the big bang created a positive vacuum energy with and energy density $>(\mathrm{GeV})^{4}$. Rather amusingly our predictions for the nature of dark matter are ipso facto completely consistent with the observed inhomogeneity of matter at practically all scales. Indeed the actual state of the art for numerical simulation of the evolution of dark matter structures use point particles with a fixed mass typically in the range $10^{3}-10^{4} M_{\odot}$ (for a review see [27] [28]). Furthermore in order to simulate the formation of galactic structures within the framework of the numerical models for the evolution of dark matter structures it is necessary to add primordial seed masses of about $10^{5} M_{\odot}$ in order to obtain the observed galactic morphologies [28]. Of course it follows from our prediction that the dark matter compact objects were formed from the stochastic coalescence of primordial dark energy stars with a mass $M^{*}$ that compact objects with mass greater than $M_{D M}$ were also formed. In summary, it appears that our explanation for the big bang can simultaneously explain the energy density, entropy, and temperature fluctuations of the CMB, as well as virtually all details of the inhomogeneous matter structures.

\section{Acknowledgements}

The authors are very grateful to T. Axelrod, P. Frampton, C. Frenk, and A. Loeb for discussions regarding observational constraints on many solar mass dark matter constituents. This work was performed under the auspices of the US Department of Energy by Lawrence Livermore National Laboratory under Contract DE-AC52-07NA27344 and was supported by the LLNL-LDRD Program under Project No. 17-ERD-120.

\section{Conflicts of Interest}

The authors declare no conflicts of interest regarding the publication of this paper. 


\section{References}

[1] Chapline, G. (2003) International Journal of Modern Physics A, 18, 3587-3590. https://doi.org/10.1142/S0217751X03016380

[2] Chapline, G. and Barbieri, J. (2014) International Journal of Modern Physics D, 23, Article ID: 1450025. https://doi.org/10.1142/S0218271814500254

[3] Chapline, G., Hohlfeld, E., Laughlin, R. and Santiago, D. (2001) Philosophical Magazine $B, \mathbf{8 1}, 235-254$. https://doi.org/10.1080/13642810108221981

[4] Chapline, G. (2005) Dark Energy Stars. Proceedings of the 22nd Texas Conference on Relativistic Astrophysics, Stanford, CA, 12-17 December 2004, 1-4.

[5] Kramer, D., Stephani, H., MacCallum, M. and Heralt, E. (1980) Exact Solutions of Einstein Field Equations. Cambridge University Press, Cambridge.

[6] deSitter, W., Edditington, A.S. and Lemaitre, G. (1931) Nature, 127, 704.

[7] Lemaitre, G. (1931) Monthly Notices of the Royal Astronomical Society, bf91, 493.

[8] Harrison, E.R. (1967) Monthly Notices of the Royal Astronomical Society, 137, 69-79. https://doi.org/10.1093/mnras/137.1.69

[9] Chapline, G. (2010) Dark Energy Stars and the Cosmic Microwave Background. ArXiv:1004.0406.

[10] Feynman, R.P. (2003) Lectures on Gravitation. Westview Press, Boulder.

[11] Joshi, P. (2007) Gravitational Collapse and Spacetime Singularities. Cambridge University Press, New York. https://doi.org/10.1017/CBO9780511536274

[12] Mazur, P.O. and Mottola, E. (2004) Proceedings of the National Academy of Sciences of the United States of America, 101, 9545-9550. https://doi.org/10.1073/pnas.0402717101

[13] Mazur, P.O. and Mottola, E. (2015) Classical and Quantum Gravity, 32, Article ID: 215024. https://doi.org/10.1088/0264-9381/32/21/215024

[14] Chapline, G. and Mazur, P.O. (2007) A Two-Phase Model for Spacetime. 23rd Pacific Coast Gravity Meeting, Pasadena, CA, 16-17 March 2007.

[15] Alcock, C., et al. (1998) The Astrophysical Journal, 499, 19.

[16] Chapline, G. and Barbieri, J. (2018) Letters in High Energy Physics, 1, 17-20. https://doi.org/10.31526/LHEP.1.2018.04

[17] Harrison, E.R. (1970) Physical Review D, 1, 2726-2730. https://doi.org/10.1103/PhysRevD.1.2726

[18] Zeldovich, Y.B. (1970) Astronomy \& Astrophysics, 5, 84-89.

[19] Peebles, P.J.E. and Yu, J.T. (1970) Astrophysical Journal, 162, 815. https://doi.org/10.1086/150713

[20] Lifshitz, E.M. (1946) Journal of Experimental and Theoretical Physics, 16, 587-602.

[21] Chapline, G. (1975) Nature, 253, 251-252. https://doi.org/10.1038/253251a0

[22] Frampton, P. (2009) Journal of Cosmology and Astroparticle Physics, 2009, 16. https://doi.org/10.1088/1475-7516/2009/10/016

[23] Clesse, S. and Garcia-Bellido, J. (2015) Physical Review D, 92, Article ID: 023524. https://doi.org/10.1103/PhysRevD.92.023524

[24] Chapline, G. and Frampton, P.H. (2016) Journal of Cosmology and Astroparticle Physics, 11, 916. https://doi.org/10.1088/1475-7516/2016/11/042

[25] Carr, B., Kuhnel, F. and Sandstad, M. (2016) Physical Review D, 94, Article ID: 083504. https://doi.org/10.1103/PhysRevD.94.083504 
[26] Ibanez, L.E. and Uranga, A.M. (2012) String Theory and Particle Physics. Cambridge University Press, New York.

[27] Frenk, C.S. and White, S.D.M. (2012) Annalen der Physik, 524, 507-534. https://doi.org/10.1002/andp.201200212

[28] Schaye, J., et al. (2012) Monthly Notices of the Royal Astronomical Society, 446. 